\title{
Studies on the Fine Structure of Wet Human Chromosome Fibers Unstained with No Fixative by Atomic Force Microscopy in Comparison with X-ray Contact Microscopy
}

\author{
Yasuhito Kinjo ${ }^{1}$, Masaji Shigeno ${ }^{2}$, Kunio Shinohara ${ }^{3}$ and Makoto Watanabe ${ }^{4}$ \\ ${ }^{1}$ Tokyo Metropolitan Isotope Research Center, Tokyo 158, Japan \\ ${ }^{2}$ Seiko Instruments Inc., Chiba 563, Japan \\ ${ }^{3}$ Faculty of Medicine, Tokyo University, Tokyo 113, Japan \\ ${ }^{4}$ Kobe City College of Nursing, Kobe 651-21, Japan
}

Accepted July 31, 1996

Eukaryotic chromosome is known to have hierarchical structures from nucleosome level to chromatid level (Watson et al. 1987). Among those structures the manner how nucleosomal chain constructs higher order fiber including " $30 \mathrm{~nm}$ fiber" has still been in controversy (Van Holde et al. 1995). A simple solenoid model (Finch and Klug 1979), where a nucleosomal chain coils syrindrically into a thicker fiber (about $30 \mathrm{~nm}$ in diameter), has generally been adopted in most current textbooks. On the other hand, there have been two major alternative models as follows: (1) Superbeads model (Hozier et al. 1977) where linearly arranged globular clusters of nucleosomes (superbeads) form the thick, condensed chromatin fiber and (2) Helical ribbon model (Woodcock et al. 1984) where coiling of condensed zig-zag array of nucleosomes (helical ribbon) generates the $30 \mathrm{~nm}$ fiber.

In addition to various physical methods such as neutron scattering, X-ray diffraction and electric dichroism, electron microscopy has long played a major role in studying the fine structure of chromosome and chromatin.

However, damages of the specimen by heat or by electron beams, desiccation of the specimen by vacuum, and in most cases, the effects of chemical treatment of the specimen (fixation and staining) have basically been inevitable and unignorable problems in conventional electron microscopy. These are all the factors which may alter the fine structures of the specimen from those in their natural cellular environment.

There have mainly been two different ways to overcome or to mitigate those problems of electron microscopy. One way has been to develop new specimen preparation techniques which are considered to maintain the fine structures of the specimen as natural as possible. To date, a number of techniques on this subject such as critical point drying method have been developed. However, there still remains a question whether the observed images correctly reflect their real structures in situ or not.

The another way has been to develop new microscopies which allow the observation of wet specimens neither fixed nor stained. Recently, we reported elsewhere (Shinohara et al. 1990, 1992, Kinjo et al. 1994) that various hierarchical structures of unfixed, unstained and hydrated human chromosome fibers including individual nucleosomes (about $12 \mathrm{~nm}$ in diameter) could be observed by soft X-ray contact microscopy (XRCM) in combination with transmission electron microscope (TEM) applying plazma-polymerization replica method (Tanaka and Karasaki 1983).

$\mathrm{X}$-ray microscopy (XRM) is one of imaging systems which records the distribution of the intensity of X-rays penetrated through the specimens. The principle of XRM is based on the 
difference in the X-ray absorption to a different element or a different moleclule. Especially in a special wavelength range $(2.3-4.4 \mathrm{~nm})$ of X-rays, namely, 'water window', there exist a big difference in the X-ray absorption between nucleic acids or proteins and water (Kirz and Sayre 1980). The difference gives contrast to images of wet biological specimens without staining. In addition, the resolution of XRM is higher than that of light microscopy because the wavelength of X-rays is shorter than that of visible light. Moreover, higher penetration depth of X-rays than that of electrons allows XRM easily to observe thick and/or wet specimens.

Our data suggested that unfixed and unstained chromatin fibers in a hydrated condition are basically composed of multiples of nucleosomes (supranucleosomal particles or superbeads, Zentgraf and Franke 1984).

However, X-ray images of relatively 'thick' specimens including metaphase chromosomes often look quite complicated since they are produced after penetration of X-rays through multiple or piled up structures of the specimens. In addition, clear images can be obtained only for the portions of the specimen tightly attached to the surface of an X-ray resist in case of contact method (Kinjo et al. 1994). The images of the portions floating in a water layer became less clear depending on the distances of those portions from the resist surface because of penumbral blurring and Fresnel diffraction. Therefore, comparative studies on the specimens prepared in the same way by XRCM and different type of microscopies which allow the observation of biological specimens as intact as possible may provide us useful information on the fine structures such as the continuity of the structures or three dimensional organization of the specimen.

Atomic force microscope (AFM) developed by Binnig et al. (1986) is one of scanning probe microscope (SPM) families. This microscope has recently been coming into wide use for the observation of various biological specimens because it can image them in a physiological

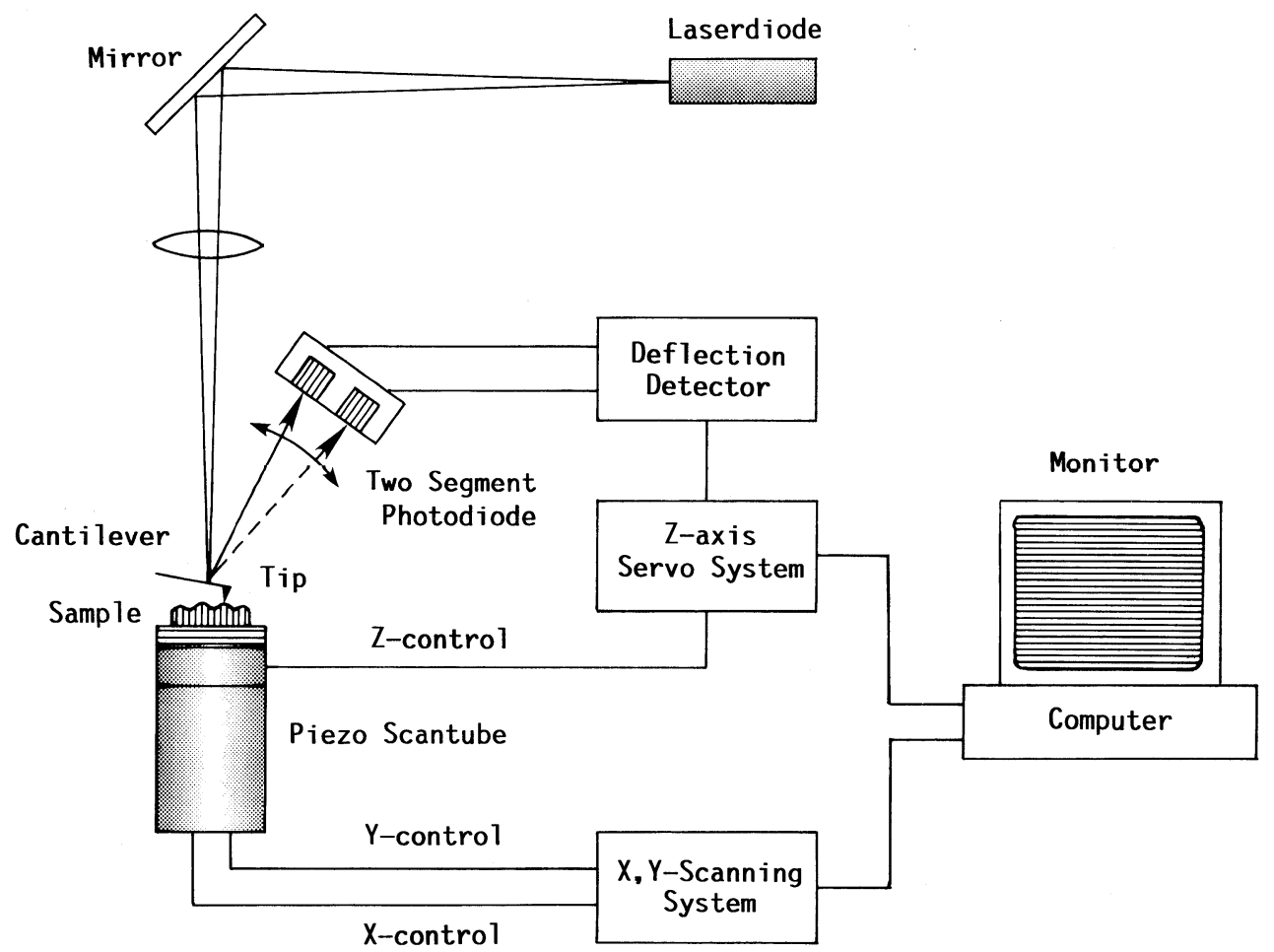

Fig. 1. Operating principle of AFM. For explanation in detail, see introductory chapter of the text. 
environment at a resolution similar to that obtained for the electron microscope (Lal and John 1994). The additional advantages of AFM are as follows: (1) Ability to observe a specimen in various (vacuum, air, gaseous or fluid) environments, (2) No requirement of any pretreatment of the specimen prior to observation, (3) Much less or few damage of the specimen during observation, (4) Measurability of various physical or physicochemical parameters such as friction force, electric potential and visco-elastisity of the specimen surface, (5) Capability of image processing and analysis, and (6) Capability of micromanipulation of the specimens.

AFM is a microscope that visualize the fine structures of specimen surface by detecting the change in interaction force (attraction or repulsion) bewteen atoms of a sharpened probe tip (tip radius of about $10 \mathrm{~nm}$ ) and those of the specimen surface during scanning the specimen surface with a tip. Fig. 1 shows the schematic illustraction of AFM. As the tip scans the specimen surface, the cantilever deflects by the interaction force between the tip and the specimen surface. The spot of the laser beam emmited onto and reflected from the back side of the cantilever is changed according to the deflection of the cantilever, and is detected by the quarterly position-sensitive photodetectors. Output of these detectors changes the feedback current of the piezo scanner to keep the deflection of the cantilever constant while the tip scans the specimen. Finally, the feedback current is computer-processed to generate a topographycal image of the specimen surface.

We have applied AFM to wet human chromosome and chromatin prepared by surfacespreading whole-mount technique (Gall 1963, Watanabe and Tanaka 1972). The same specimen preparation technique has been used by us also for EM and XRCM. In this communication, we report the results of comparatiave study by AFM and XRCM on the fine structures of wet human chromosome/chromatin neither fixed nor stained.

Materials and methods

\section{Cell culture}

Human lymphocyte cell line (RPMI 1788) were purchased from GIBCO and has been maintained at $37^{\circ} \mathrm{C}$ in RPMI 1640 medium supplemented with $10 \%$ fetal bovine serum, 2 $\mathrm{mg} / \mathrm{ml}$ sodium bicarbonate and $0.1 \mathrm{mg} / \mathrm{ml}$ streptomycin sulfate (Meiji Confectionery Co.) plus 100 units/ml penicillin G-potassium (Meiji Confectionery Co.) in humidified 5\% $\mathrm{CO}_{2}-95 \%$ air.

To obtain images of mitotic chromosomes, cells in logarhythmic growth phase were partially synchronized to M-phase by the treatment with $0.05 \mu \mathrm{g} / \mathrm{ml}$ colcemid (Wako Pure Chemicals Co.) for $16 \mathrm{hr}$.

\section{Preparation of chromosomes/chromatin (Surface-spreading whole-mount technique) for XRCM and $A F M$}

The procedure is schematically shown in Fig. 2. Small amount of the cells centrifuged at $1000 \mathrm{rpm}$ for $5 \mathrm{~min}$ were placed with a small (less than $1 \mathrm{~mm}$ in diameter) platinum loop on a clean surface of pure water. At this moment the intracellular chromosomes/chromatin were released from the burst cells, and were spread as a thin film over the water surface. The specimen substrate horizontally held by a holder was lightly touched to the surface. Then the surface film was attached to a clean surface of PMMA (polymethylmethachlyrate: X-ray resist) supported by a silicon substrate in case of XRCM. While in case of AFM, the surface film was transferred in the same way to the surface of various substrates such as glass, silicon, graphite or PMMA. 

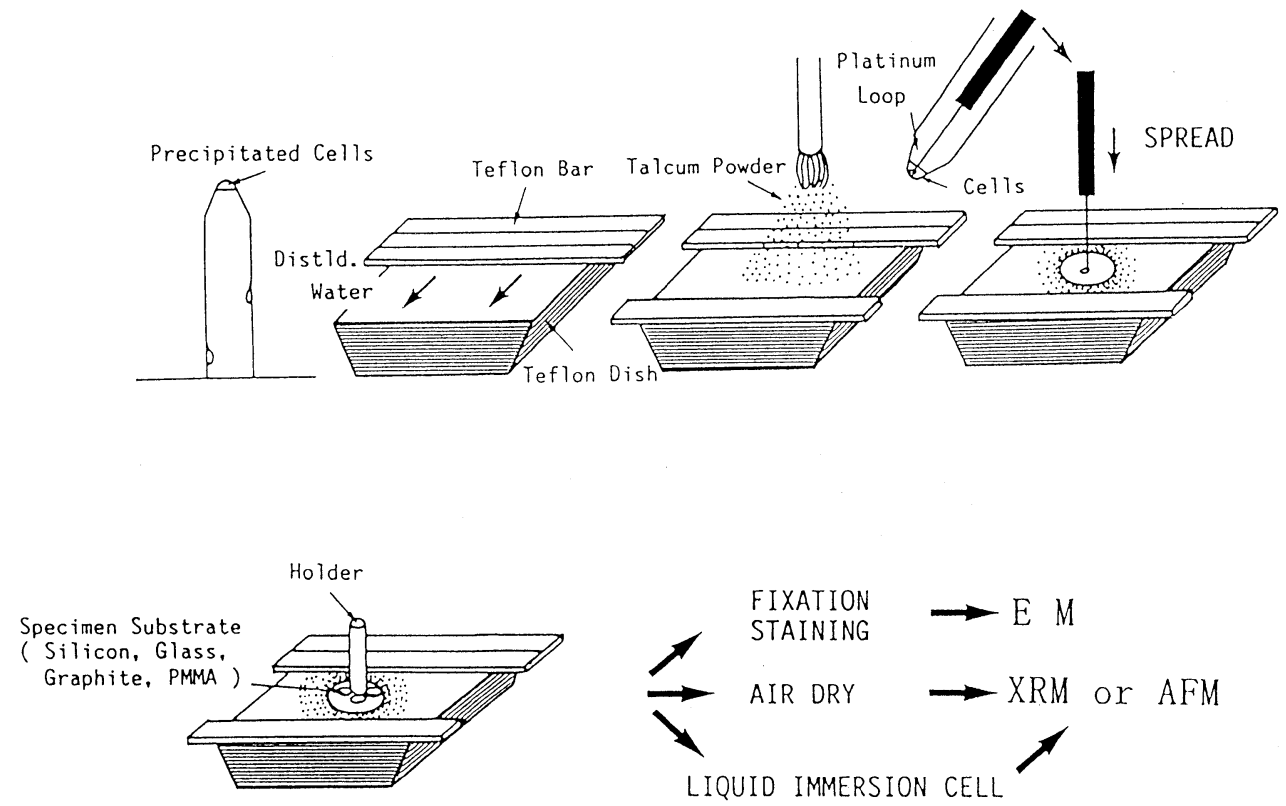

Fig. 2. Procedure of surface-spreading whole-mount technique.

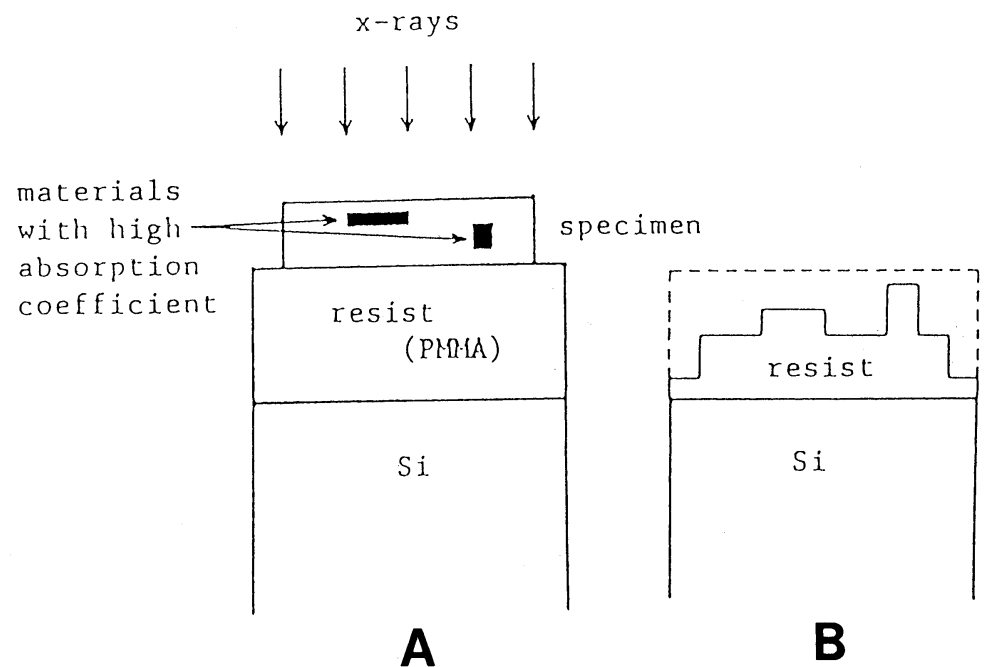

Fig. 3. Principle of X-ray contact microscopy: X-ray resist (PMMA) is exposed to X-rays through materials tightly attached to the surface of the resist (A) and developed after X-ray exposure (B).

procedures from X-ray exposure of the resist with specimens to observation of the replica of the chemically etched (developed) resist after X-ray exposure are described in detail elsewhere (Kinjo et al. 1994). In brief, a resist (PMMA supported by a silicon substrate) with unfixed and unstained chromosomes/chromatin in a fluid environment was mounted in a simple, air-tight chamber with a silicon nitride window (Fig. 4, Shinohara et al. 1992), exposed to laser-produced plasma X-rays and developed with the mixture of methylisobutylketone and isopropanol after removing the materials from the resist surface with $0.5 \%$ sodium hypochlorite. A replica film of the developed resist was made by plasma-polymerization replica method (Tanaka 1983) and was observed in a TEM. 
A
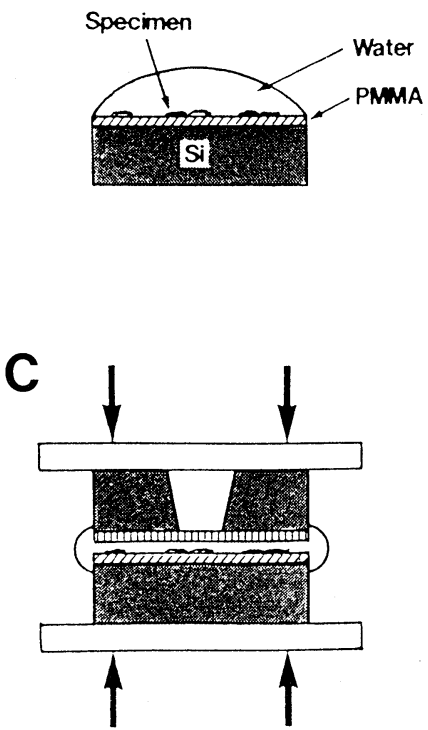

B

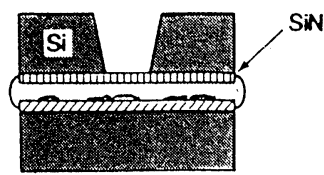

D

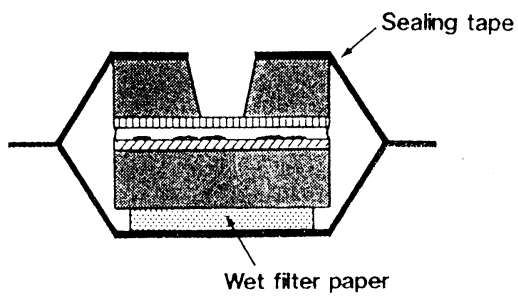

Fig. 4. Procedure for mounting specimen with water in an air-tight chamber for X-ray contact microscopy (Kinjo et al. 1994): The water-spread specimens are attached with water to the surface of PMMA supported on a silicon wafer (A). The wet specimens are then covered with silicon nitride window (B) and press-fitted together with torque gauge (C). This assembly is sealed with two pieces of adhesive tape, together with a wetted filter paper (D).

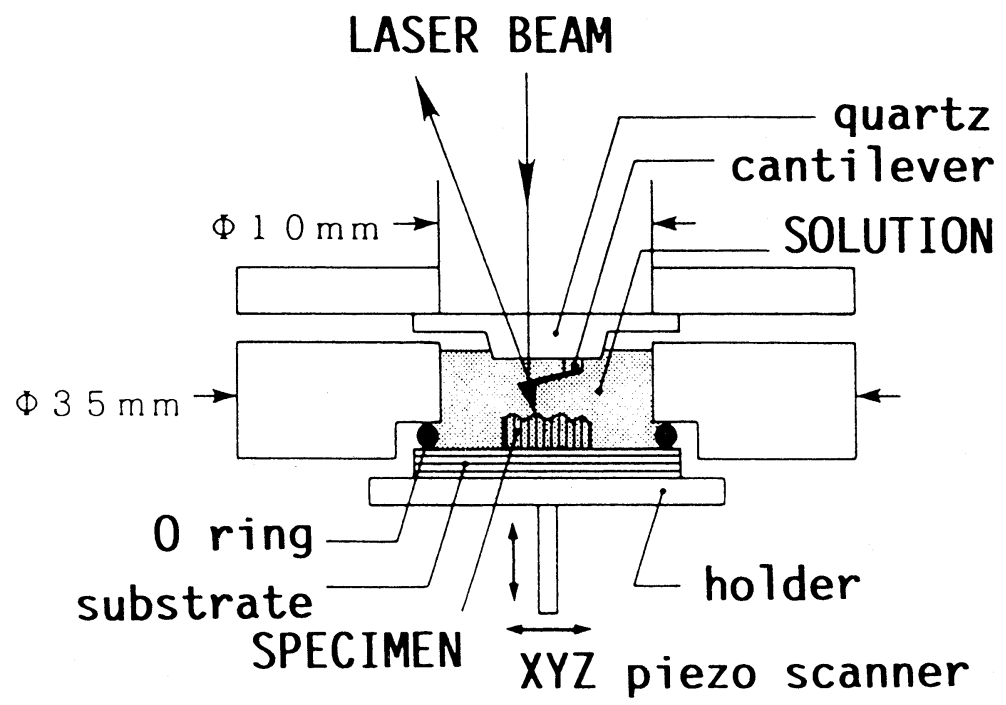

Fig. 5. Liquid immersion cell for AFM: The specimen tightly sealed in a fruid environment is scanned with a probe tip integrated with a cantilever. The laser beam reflected from the back side of a cantilever is detected through a quartz window and computer-processed to produce an image.

\section{AFM observation}

Immediately after preparing the surface film onto a substrate, excess water was removed with a piece of a filter paper. Then the substrate on which the spread chromosomes/chromatin were embedded was mounted in a liquid immersion cell (Fig. 5, Watanabe et al. 1994) of AFM 
(SEIKO SPI 3700) with serum-free culture medium. AFM observation was carried out by operationg a cantilever with an integrated pyramidal tip (tip radius of about $10 \mathrm{~nm}$ in diameter) made of silicon nitride at a force less than $10^{-9} \mathrm{~N}$. The monitored topographic images were computer-processed (tilted) to produce pseudo-three dimensional images. In some cases, image
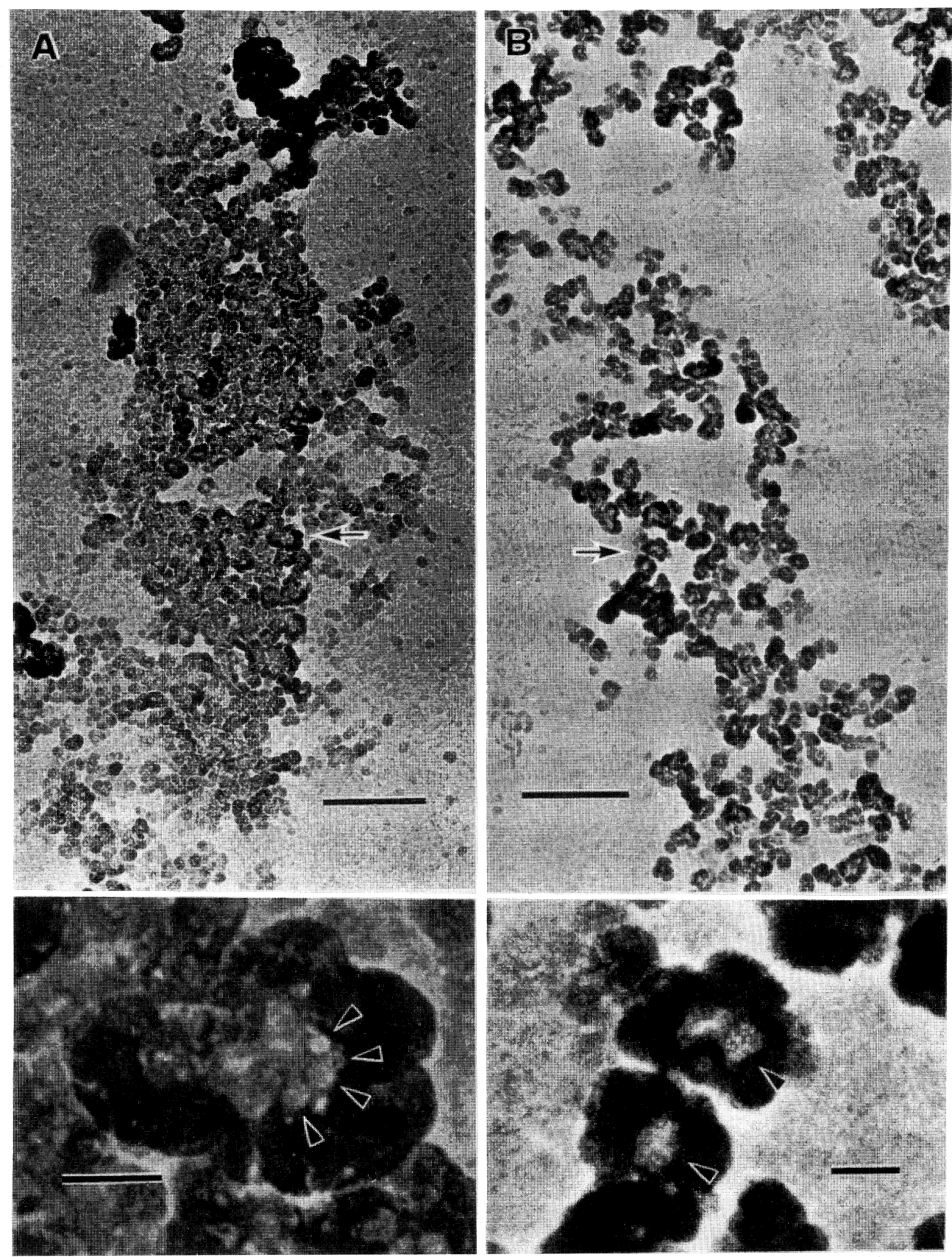

Fig. 6. X-ray images of wet human chromosome/chromatin fibers neither fixed nor stained: condensed fiber (A) and decondensed fiber (B). The lower pictures are magnified views of the upper pictures where moluroid organization of nucleosomes (seen as globular clusters of nucleosomes: superbeads; arrowheads) is evident. The bars represent $1 \mu \mathrm{m}$ (upper pictures) and $100 \mathrm{~nm}$ (lower pictures), respectively. 
analyses such as cross sectional prifile analysis and size measurement of particls or fibers were carried out.

\section{Results}

Fig. 6 (reconstructed from Kinjo et al. 1994) shows X-ray images of condensed (A) and decondensed (B) wet chromatin neither fixed nor stained. The actual images of the specimens are represented as white areas covered with dark areas. Complicated fibrous network with "knobby" fibers are evident. The lower pictures are magnified views of the arrowed areas of the upper pictures where globular clusters of nucleosome-like particles (arrowheads: "moluroid organization", Zentgraf and Franke 1984) are evident.

Fig. 7 shows an AFM image of single wet human chromosome neither fixed nor stained. A fiber with also typical knobby appearance (small arrows) stretches out from the periphery of each chromatid. A loop structure of a fiber is seen at a portion indicated by a larger arrow.

Fig. 8 shows a magnified view of the arrowed area in Fig. 7. Various sizes of particles constructing a fiber and those around a fiber are evident. Especially at the portion indicated by arrowheads, particles with the sizes ranging about $50-60 \mathrm{~nm}$ in diameter can clearly be identified as components of a fiber.

Fig. 9 shows an example of cross-sectional analysis of an obtained image. The graph of the lower left in this figure shows a cross-sectional profile of the portion indicated by a straight line across a stretched fiber and a particle of the picture (almost the same area as Fig. 8) in the right rectangle. The distances of a fiber and a particle were roughly estimated to be about 40 and 50 $\mathrm{nm}$, respectively.

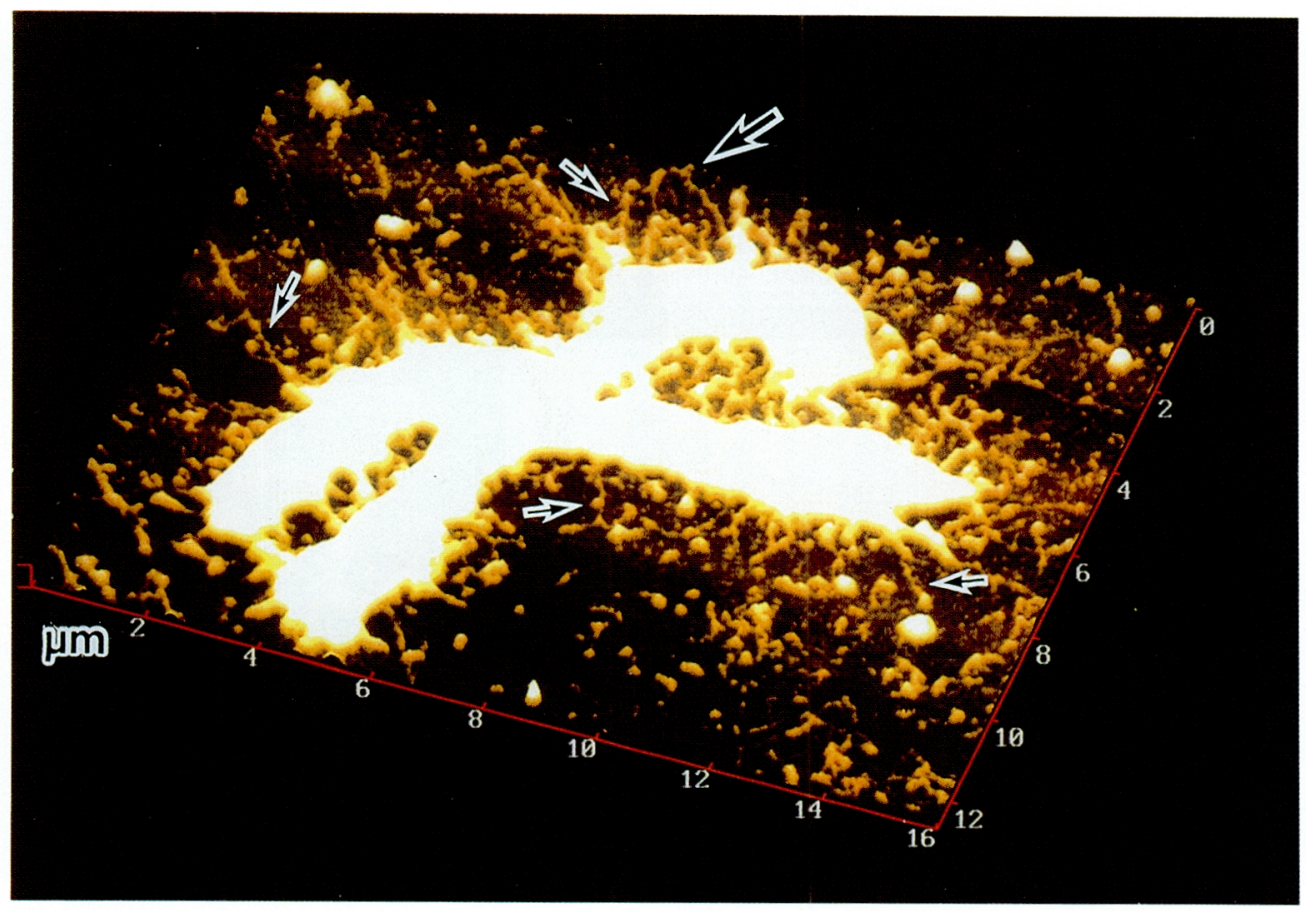

Fig. 7. AFM image of single human chromosome in solution neither fixed nor stained. Note the looping fiber unravelled from the periphery of one arm (arrow). 


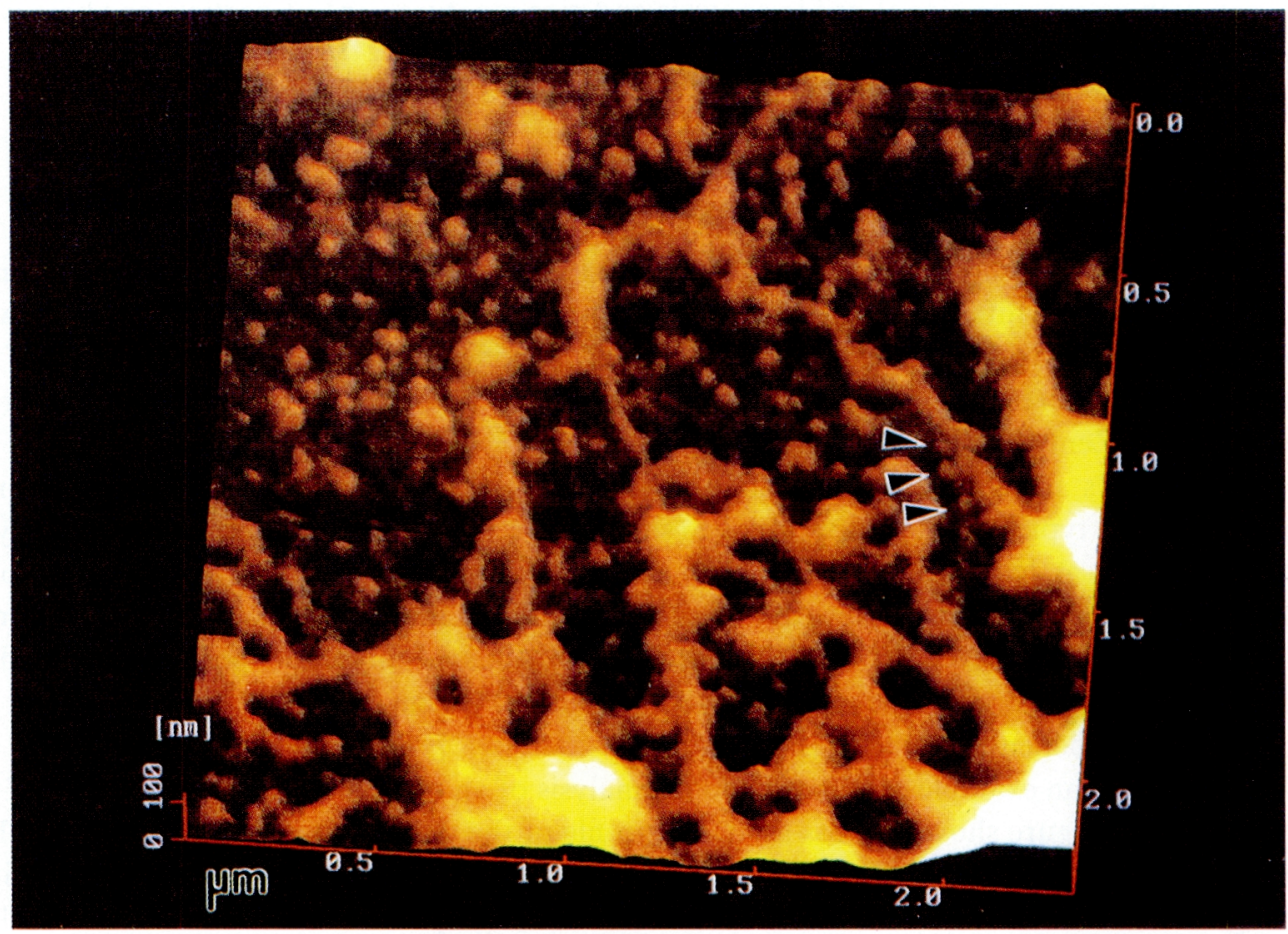

Fig. 8. Magnified view of an arrowed area in Fig. 1. Note the fiber with typical knobby appearance and superbeads-like particles within and around the fiber.

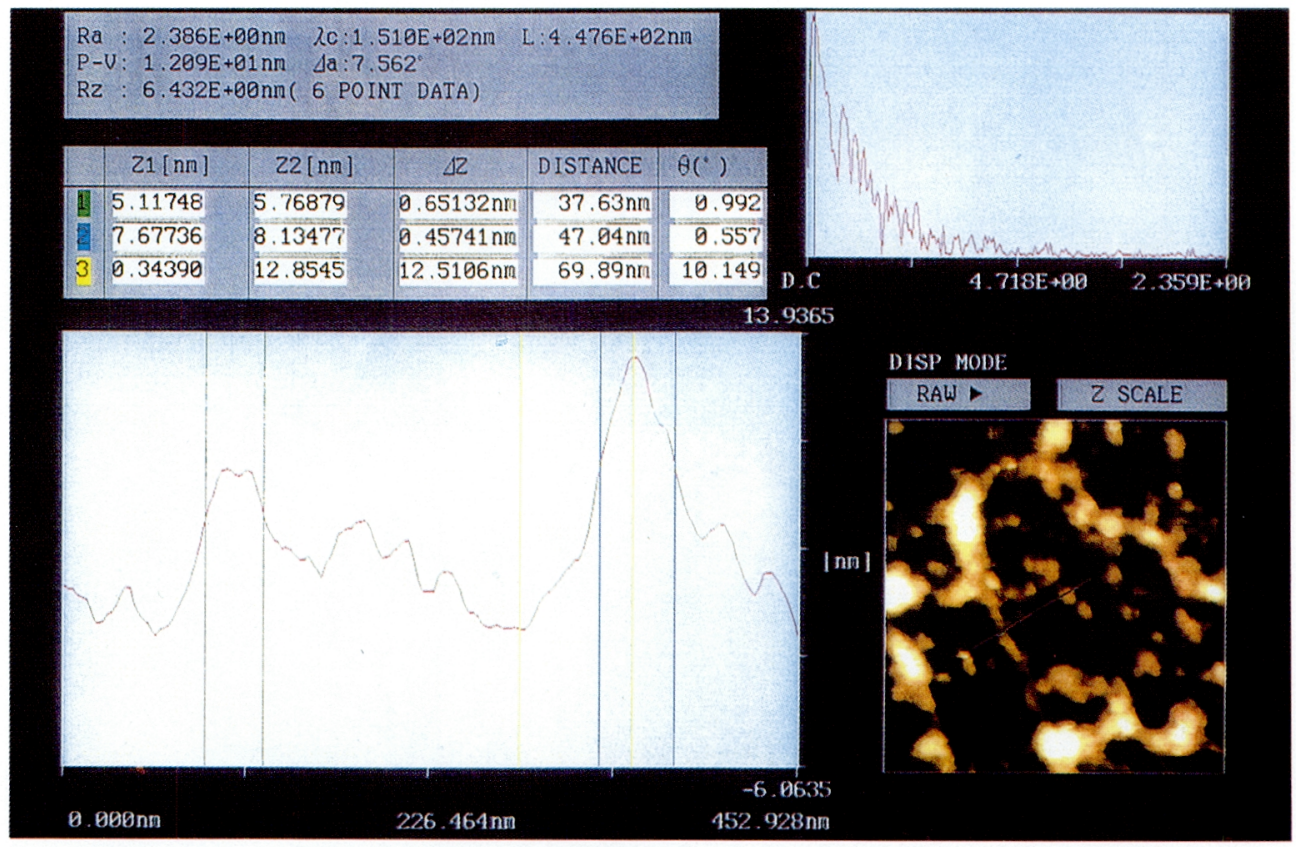

Fig. 9. Cross-sectional profile and the estimated widths and heights of a fiber and a particle. 


\section{Discussion}

In the present study, it has been demonstrated that fine structures of wet human chromosome/chromatin neither fixed nor stained can be observed by AFM. At first we were afraid that it was impossible to observe the chromosome/chromatin specimens in solution because most of them might come off from the surface of a substrate during scanning of them with a probe tip. However, they remained attaching to the substrate surface at least during observation, allowing us to observe their fine structures. Especially in the peripheral areas of a chromosome, fibers with typical knobby appearance presumably representing in size and shape that chromatin fibers are basically composed of multiples of nucleosomes (supranucleosomal particles or superbeads) were evident.

Individual nucleosomes in superbeads ('moluroid' organization, Zentgraf and Franke 1984) which could have been visible in X-ray images (Fig. 6, Kinjo et al. 1994) could hardly be identified in the present study. This may be attributed to the following factors: (1) The shape (irregularity at atomic scale) and/or the radius of the individual probe tip used, (2) Dragging of the rough and soft surface of the specimen with the tip, and (3) Convolution of the probe tip's shape with the actual shape of the specimen. Allen et al. (1992) described that lateral resolution of AFM was lower than $10 \mathrm{~nm}$ when using commercially available probe tips. Lal and John (1994) also described that the resolution of AFM is relatively poor $(\sim 10 \mathrm{~nm})$ when observing biological specimens in comparison with crystalline solid specimens or many inorganic materials $(\sim 0.1-0.2 \mathrm{~nm})$. However, in case of highly stretched chromatin fibers of rDNA minichromosomes of Tetrahymena, individual nucleosome cores arranged in a zig-zag conformation could be observed with AFM (Martin et al. 1995).

Long fibrous structure of chromatin including chromatin loops could be observed more clearly by AFM than by XRCM. In case of XRCM, clariness of the image depended on the distance between the resist surface and the spot of the specimen as described in the introductory chapter.

On the other hand, AFM can clearly image everywhere of the specimen surface regardless of a little difference in the distance of the spot of the specimen from the substrate surface as far as the probe tip can trace it.

Special emphasis should be given to the usefulness of AFM that clear images including three dimensional representation of human chromosome/chromatin with sufficient contrast could easily be observed by AFM without any pretreatment of the specimen.

It was demonstrated that the present results were basically compatible with those obtained by XRCM as expected. At the same time, it was also demonstrated in the present study that both methods (XRCM and AFM) can provide complementary information each other on the fine structure of chromosome/chromatin at least for resolution and image continuity.

On the limited resolution of AFM in observing rough surface of 'soft' specimens like wet chromosome/chromatin, some improvements of the apparatus have recently been in progress. One of them is DFM (Dynamic Force Mode)-in-solution unit with a specially designed tip developed by SEIKO Instrument Inc. The unit allows one to image 'soft' specimens at high resolution without dragging the surface of the specimen with a probe tip by controlling the tip-specimen force between non-contact mode and 'cyclic contact' mode. With this unit we are trying to image more detailed structures of chromosome/chromatin including internal structure of superbeads in a condition as physiological as possible. In addition, analyses of the realtime changes of those structures accompanied with various functional changes in cells such as radiation damage, programmed cell deth (apoptosis) and carcinogenesis are our next aim. 


\section{Summary}

The fine structure of wet human chromosome/chromatin neither fixed nor stained was studied by atomic force microscopy (AFM) in comparison with soft X-ray contact microscopy (XCRM). Fibers with typical knobby appearance and particles of $30-60 \mathrm{~nm}$ in diameter within and around the fibers were evidently observed by AFM. Globular clusters of nucleosomes ('moluroid' organization of nucleosomes, superbeads) were observed by XCRM in the specimens prepared in the same way.

Both data suggested that chromatin fibers are basically composed of various sizes of condensed particles.

\section{References}

Allen, M. J., Hud, N. V., Balooch, M., Tench, R. J., Siekhaus, W. J. and Balhorn, R. 1992. Tip-radius-induced artifacts in AFM images of protamine-complexed DNA fibers. Ultramicroscopy 42-44(Pt.B): 1095-1100.

Finch, J. T. and Klug, A. 1976. Solenoid model for superstructure in chromatin. Proc. Nat. Acad. Sci. USA. 73: 18971901.

Gall, J. 1963. Chromosme fibers from an interphase nucleus. Science 139: 120-121.

Hozier, J. Renz, M. and Nehls, P. 1977. The chromosome fiber: Evidence for an ordered superstructure of nucleosomes. Chromosoma (Berl.) 62: 301-317.

Kinjo, Y., Shinohara, K., Ito, A., Nakano, H., Watanabe, M., Horiike, Y., Kikuchi, Y., Richardson, M. C. and Tanaka, K. A. 1994. Direct imaging in a water layer of human chromosome fibres composed of nucleosomes and their higher-order structures by laser-plasma X-ray contact microscopy. J. Microsc. 176(Pt.1): 63-74.

Kirz, J. and Sayre, D. 1980. Soft X-ray microscopy of biological specimens. In Synchrotron Radiation Research. Winnick, H. and Doniach, S., Editors. Plenum Press, Inc., New York. 277-318.

Lal, R. and John, S. A. 1994. Biological applications of atomic force microscopy. Am. J. Physiol. 266 (Cell Physiol. 35): $\mathrm{C} 1-\mathrm{C} 21$.

Martin, L. D., Vesenka, J. P., Henderson, E. and Dobbs, D. L. 1995. Visualization of nucleosomal substructure in native chromatin by atomic force microscopy. Biochemistry 34: 4610-4616.

Shinohara, K. 1990. Application of X-Ray microscopy to mammalian cells. In X-Ray Microscopy in Biology and Medicine. ed. by Shinohara, K. et al., Japan Sci. Soc. Press. Tokyo/Springer-Verlag, Berlin, pp. 203-212.

-, Nakano, H., Kinjo, Y. and Watanabe, M. 1990. Fine structure of unstained human chromosome fibres dried with no fixative as observed by X-ray contact microscopy. J. Microsc. 158(Pt.3): 335-342.

-, Ito, A., Kinjo, Y., Watanabe, M., Kikuchi, Y. and Tanaka, K. A. 1992. A simple chamber for hydrated specimens in a vacuum and its application to X-ray contact microscopy with laser-produced plasma X-Rays. In Springer Series in Optical Sciences. Vol. 67. X-Ray Microscopy Eds. Michette, A., Morrison, G. and Buckley, C. Springer-Verlag Berlin Heidelberg. 347-349.

Tanaka, A. 1983. A new replica method for electron microscopic studies with plasma polymerization film in a glow discharge. Seikagaku (Biochemistry) 55: 1212-1219 (in Japanese).

Van Holde, K., Zlatanova, J., Arentz, G. and Moudrianakis, E. 1995. Elements of chromatin structure: histone, nucleosomes, and fibres. Frontiers in Molecular Biology 9; Chromatin Structure and Gene Expression: pp. 126. (ed. by Elgin, S. C. R., IRL Press, Oxford).

Watanabe, M. and Tanaka, N. 1972. Supertwisting organizations of DNA molecules and their rheological properties in chromosomes and nuclei. Jpn. J. Genet. 47: 1-18.

-, Kinjo, Y. and Shigeno, M. 1994. Observation of human chromosomes by atomic force microscopy in comparison with electron microscopy. Proc. 13th. Internatl. Cong. Electron Microscopy. Vol. 3A. 445-446.

Watson, J. D., Hopkins, N. H., Roberts, J. W., Steitz, J. A. and Weiner, A. M. 1987. Molecular Biology of the Gene, 4th ed. Vol. 1: The Benjamin/Cummings Publishing, Menlo Park, California pp. 676-744.

Woodcock, C. L. F., Frado, L. L. Y. and Rattner, J. B. 1984. The higher order sturcture of chromatin: evidence for a helical ribbon arrangement. J. Cell Biol. 99: 42-52.

Zentgraf, H. and Franke, W. 1984. Differences of supranucleosomal organization in different kinds of chromatin: cell type-specific globular subunits containing different number of nucleosomes. J. Cell Sci. 99: 272-286. 